\title{
Kooperationsbeziehungen deutscher Klein- und Mittelunternehmen in Mittel- und Osteuropa
}

\author{
Ralph-Elmar Lungwitz \\ Manfred Wannöffel \\ Yvonne Rückert
}

Nach 1990 haben Kleine und Mittlere Unternehmen (KMU) aus Westeuropa und insbesondere aus Deutschland die Transformationsländer Mittel- und Osteuropas in zunehmendem Maße in die Reorganisation ihrer Wertschöpfungsketten einbezogen. Obwohl die Auswirkungen dieser Prozesse wahrscheinlich mehr Menschen betrafen als die Investitionen großer Konzerne, standen sie weit weniger im Blickpunkt der Öffentlichkeit. Der Beitrag geht anhand der Situation in zwei Branchen der Frage nach, welche Konsequenzen sich aus den dominierenden Mustern der Arbeitsteilung zwischen deutschen KMU und ihren Partnerfirmen für die Beschäftigten in Mittel- und Osteuropa ergeben.

\section{Kooperationsbeziehungen von KMU und die europäische Arbeitsteilung}

Aus dem grundsätzlichen Bekenntnis der Europäischen Union (EU) zu einer Konvergenz der Lebensbedingungen in ihren Mitgliedsländern ergibt sich eine besondere Priorität für die Transformationsländer in Mittel-, Ost- und Südosteuropa, von denen erwartet wird, dass sie in einem Prozess nachholender Modernisierung und überdurchschnittlichen Wachstums ihre Entwicklungsrückstände gegenüber den westeuropäischen Kernstaaten der EU allmählich verringern und perspektivisch ganz überwinden. In der Tat konnten die mittel-/ osteuropäischen EU-Staaten in den letzten Jahren ein relativ hohes Wirtschaftswachstum und eine Steigerung der Löhne erreichen. Setzt man das Lohnniveau 1995 gleich 100, so erhöhten sich die Nominallöhne in den neuen EU-Staaten bis 2004 auf den Faktor 251 (Schulten 2004). Allerdings kann sich die Bestimmung der Konvergenz nicht in der allmählichen Annäherung auf der Ebene von makroökonomischen Kennziffern erschöpfen. Von entscheidender Bedeutung sind die sich dahinter verbergenden Differenzen der tatsächlichen Lebensbedingungen.

Bei der Arbeitsteilung zwischen deutschen KMU und ihren Partnerfirmen in Mittel- und Osteuropa handelt es sich letzten Endes um einen spezifischen Teilaspekt einer komplexeren Frage, und zwar der nach den dominanten Mustern der Arbeitsteilung zwischen den westeuropäi- schen „Altmitgliedern“ der EU und den neuen Mitgliedstaaten aus Mittel-/Osteuropa und ihren Folgen für die Lebensbedingungen der Beschäftigten. Sollte diese Arbeitsteilung dauerhaft darauf basieren, dass wertschöpfungsintensive Prozessphasen und strategische Funktionen wie Produktentwicklung und Vertrieb den deutschen Unternehmen vorbehalten bleiben, während die mittel-/osteuropäischen Partnerfirmen im Wesentlichen als verlängerte Werkbänke agieren, dürfte das Ziel einer Konvergenz von Wirtschaftsleistung und Wohlstand zwischen den alten und den neuen Mitgliedern der EU kaum realisierbar sein.

\section{Grundlagen und Rahmen- bedingungen}

Im Folgenden gehen wir in einem zwar begrenzten, aber dennoch keinesfalls unwichtigen Teilbereich grenzüberschreitender wirtschaftlicher Austauschprozesse, den Fragen nach, wie die Beschäftigungsbedingungen und Partizipationsformen in mittel-/osteuropäischen Unternehmen, die mit deutschen KMU kooperieren, beschaffen sind, und wie dies mit der Struktur der Arbeitsteilung zwischen den Partnern zusammenhängt. ${ }^{1}$ Es gibt zwar vielfältige Debatten, theoretische Konzepte und empirische Untersuchungen zu Wertschöpfungsketten, Netzwerken etc. - auch unter Einschluss Mittel-/Osteuropas -, jedoch wurden dabei, abgesehen von pauschalen Hinweisen auf die geringen Arbeitskosten, die Beschäftigungsbedingungen und $\mathrm{Ar}$ - beitsbeziehungen in den einbezogenen mittel-/osteuropäischen Unternehmen kaum thematisiert. ${ }^{2}$

\footnotetext{
Dabei stützen wir uns im Wesentlichen auf die Ergebnisse eines von der Hans-Böckler-Stiftung (mit Unterstützung der IG Metall und der FriedrichEbert-Stiftung) geförderten Forschungsprojektes "Arbeits- und Beschäftigungsbedingungen in grenzüberschreitenden Wertschöpfungsketten deutscher KMU - Eine Untersuchung von Unternehmen aus den Bereichen Textil/Bekleidung und Holz/Kunststoff in Polen, Tschechien und Rumänien“. Die im Rahmen dieses Projektes erhobenen Ergebnisse wurden durch Befunde aus den Forschungsprojekten KONET (Universität Dortmund) und "Kooperationsbeziehungen ostdeutscher KMU mit Unternehmen in Mittel-/Osteuropa" (WISOC) ergänzt und teilweise validiert (s. www.konet.de; www.wisoc.de).

2 Eine Ausnahme stellen hier die von der Frauenbewegung inspirierten Analysen zu den Beschäftigungsbedingungen von Frauen in der globalisierten Bekleidungsindustrie dar (Musiolek 2006).
}

Ralph-Elmar Lungwitz, Dr., Institut für Wirtschafts- und Sozialforschung Chemnitz e. V. Arbeitsschwerpunkte: Arbeitsbeziehungen, Netzwerksforschung, Mittel-/Osteuropa. e-mail:wisoc@t-online.de Manfred Wannöffel, Dr., Gemeinsame Arbeitsstelle Ruhr-Universität Bochum IG Metall. Arbeitsschwerpunkte: Internationale Mitbestimmungsforschung, Wirtschafts- und Organisationssoziologie. e-mail: Manfred.Wannoeffel@ruhr-unibochum.de Yvonne Rückert, Dipl.-Soziologin, Gemeinsame Arbeitsstelle Ruhr-Universität Bochum - IG Metall. Arbeitsschwerpunkte: Arbeitsbeziehungen, internationale Gewerkschaftspolitik, Arbeits- und Sozialstandards. e-mail: yvonne.rueckert@rub.de 
Bei Kooperationen handelt es sich um intentional geschaffene, relativ stabile Austauschbeziehungen zwischen rechtlich und wirtschaftlich eigenständigen Unternehmen mit dem Ziel eines wechselseitigen Zugriffes auf Ressourcen. Durch diesen Zugriff auf Ressourcen des jeweils anderen Unternehmens werden Handlungsmöglichkeiten eröffnet, die bei der Beschränkung auf innerbetriebliche Potenziale unerreichbar wären, zumindest aber kurzfristig verschlossen blieben (Semlinger 1993). Die Art der Ressourcen, um derentwillen sich Unternehmen auf das durchaus riskante Unterfangen einer Kooperationsbeziehung einlassen, spiegelt sich in den jeweiligen Kooperationsmotiven der Unternehmen wider. Die Reduktion von Kosten, die Zusammenarbeit mit ausländischen Partnern zum Zwecke der MarkterschlieBung oder die Absicht, einem wichtigen Kunden zu folgen, gelten gemeinhin als vorrangige Motive für deutsche Firmen, sich im Ausland wirtschaftlich zu engagieren. Betrachtet man jedoch nur das Segment deutscher KMU und deren wirtschaftliches Engagement in Mittel-/Osteuropa, so zeigen empirische Untersuchungen, dass zwar auch hier alle diese Motive auftreten, dass aber dem Motiv der Kosteneinsparung eine eindeutige Dominanz zukommt (Bluhm 2000; Kinkel et al. 2004). Die entscheidende Ressource, auf die deutsche KMU zur Erreichung dieses Zieles zugreifen, besteht in den vergleichsweise niedrig bezahlten und dennoch qualifizierten Arbeitskräften in Mittel-/Osteuropa. Die Kooperationsbeziehungen ermöglichen es den mittel-/osteuropäischen Unternehmen, bestimmte Teilfunktionen im Wertschöpfungsprozess ihrer deutschen Partnerfirmen zu übernehmen und ihnen die produzierten Waren oder Komponenten zu Preisen zu liefern, die bei ihrer ausschließlichen Produktion in Deutschland nicht zu erreichen wären. Für die Firmen aus Mittel-/Osteuropa stellt die Zusammenarbeit mit deutschen Firmen oft die einzige Möglichkeit dar, indirekt an einem Markt partizipieren zu können, den sie aus eigener Kraft in der Regel nicht erschließen könnten.

Wenn sich deutsche Firmen dazu entschließen, eine Kooperationsbeziehung zu einem mittel-/osteuropäischen Partner einzugehen, so wird damit eine Austauschbeziehung zwischen Unternehmen etabliert, die unter immer noch recht ungleichen institutionellen, wirtschaftlichen und sozialen Rahmenbedingungen agieren. Diese Bedingungen sind, was Mittel- und Osteuropa betrifft, durch den Transformationsprozess geprägt. $\mathrm{Zu}$ ihnen gehören unter anderem ein Einkommensniveau, das immer noch signifikant unter dem deutschen liegt, das weitgehende Fehlen betrieblicher Arbeitnehmervertretungen im Bereich privater KMU sowie von überbetrieblichen Tarifverträgen und eine insgesamt ausgeprägte Innovationsschwäche. ${ }^{3}$

Es ist bekannt, dass KMU nicht nur in Mittel- und Osteuropa seltener über eine institutionalisierte Arbeitnehmervertretung verfügen als Großbetriebe (Wassermann/Rhode 2004; Gohde 2005). Sie sind auch schwächer in dem System institutioneller Regelungen vertreten, das auf die Sicherung von Arbeitnehmerinteressen im Rahmen von Globalisierungsprozessen gerichtet ist (Brandl/Stelzl 2005; Greven 2006). Dazu zählen Regelungen zu den Europäischen Betriebsräten und zur Europäischen Gesellschaft ebenso wie die Aktivitäten europäischer und internationaler Gewerkschaftsverbünde zum Abschluss von Rahmenabkommen mit Unternehmerverbänden oder mit Einzelunternehmen. Man kann also in Bezug auf KMU von einer "doppelten Vertretungslücke" sprechen, da diese nicht nur im nationalen Rahmen Defizite bei der institutionalisierten Vertretung von Arbeitnehmerinteressen aufweisen, sondern weil sie auch bei den supranationalen Regelungen zur sozialen Kontrolle von wirtschaftlichen Internationalisierungsprozessen eine deutlich schwächere Position haben als Großunternehmen.

\section{Kooperationspartner deutscher KMU in Mittel-/ Osteuropa}

Es ist schwierig, genaue Zahlen hinsichtlich der internationalen Kooperationsbeziehungen von KMU zu ermitteln, da diese, insbesondere dann, wenn keine Kapitalbeteiligung vorliegt, nicht statistisch erfasst werden. ${ }^{4}$ Dennoch lassen sowohl Experteneinschätzungen als auch statistische Indizien den Schluss zu, dass deutsche KMU ihre Kooperationsbeziehungen in die Länder Mittel-/Osteuropas seit 1990 systematisch ausgebaut haben. Die durch kleine und mittlere Unternehmen geprägten Branchen Möbel und Bekleidung stellten gewissermaßen die Klassiker einer auf Kostensenkung ausgerichteten Kooperation deutscher KMU mit Partnerfirmen in Mittel-/ Osteuropa dar. So stieg beispielsweise der Anteil von Bekleidung und Möbeln am polnischen Export von 6,7 \% im Jahre 1989 auf 17,9 \% im Jahre 1995 (Kurz/Wittke 1998), wobei der größte Teil dieser Erzeugnisse nach Deutschland geliefert wurde. Inzwischen ist der Anteil der Exporte dieser Branchen am Gesamtexport sowohl in Polen als auch in anderen mittel-/osteuropäischen Ländern rückläufig, andere Branchen wie Fahrzeug- und Maschinenbau haben an Bedeutung gewonnen. Dennoch wurden 2002 ca. $80 \%$ der Produktionskapazitäten der polnischen Bekleidungsindustrie für die Lohnveredlung, in erster Linie für deutsche Auftraggeber, eingesetzt (Peplak 2002). In den letzten Jahren hat sich Rumänien zum größten Bekleidungshersteller Europas entwickelt, $90 \%$ des Produktionsvolumens werden im Rahmen der Lohnveredlung genutzt (Preiss 2005). $80 \%$ des Umsatzes der polnischen und $60 \%$ der tschechischen Möbelindustrie ${ }^{5}$ werden über den Export realisiert, wobei die Lieferung von Komplettmöbeln und Komponenten an deutsche Partnerfirmen eine wichtige Rolle spielt.

Ungeachtet des Defizits an differenzierten statistischen Aussagen bleibt festzuhalten: Zahlreiche Unternehmen in Mittel-/ Osteuropa gründen ihre Existenz ganz oder teilweise auf Kooperationsbeziehungen zu deutschen KMU, die Lebenswirklichkeit Tausender Arbeitnehmerinnen und Arbeitnehmer in diesen Ländern ist durch die in diesen Unternehmen existierenden Beschäftigungsbedingungen und Arbeitsbeziehungen geprägt. Die Bekleidungsindustrie und die Möbelindustrie spielen in diesem Zusammenhang immer noch eine wichtige Rolle, wenn sie auch gegenüber anderen Branchen in den letzten Jahren relativ an Gewicht verloren haben. Entspre-

3 In der "Innovation Scorecard “-Untersuchung der EU nahm Tschechien den 20. und Polen den 21. Platz unter 25 Staaten ein.

4 Außerdem weist die Außenhandelsstatistik keine differenzierten Angaben nach Betriebsgrößen aus und unterscheidet nicht zwischen dem Umsatz von Tochterfirmen und Mutterfirmen oder dem Umsatz zwischen Kooperationspartnern.

5 Angaben für Polen: Gazeta Drzewna (Dezember 2004), Angaben für Tschechien: Experteninterview mit dem Vertreter des Verbandes der Tschechischen Möbelindustrie. 
chend ihrer jeweiligen Bedeutung wurden Kooperationsbeziehungen deutscher KMU in folgende Länder in unsere Untersuchung einbezogen: Für die Bekleidungsindustrie Polen und Rumänien und für die Möbelindustrie Polen und Tschechien.

Bei den mittel-/osteuropäischen Partnerfirmen, die wir unter dem Blickwinkel ihrer Kooperationsbeziehungen und der Arbeitsteilung mit deutschen Partnern untersucht haben, handelte es sich vorwiegend ebenfalls um KMU. In einigen Fällen stießen wir allerdings auch auf größere Unternehmen, die mit deutschen KMU zusammenarbeiteten.

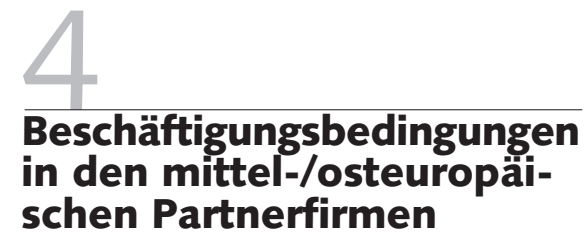

\subsection{METHODISCHE GRUNDLAGEN}

Den empirischen Kern des Forschungsprojekts bildeten jeweils sechs Fallstudien zu Kooperationsbeziehungen deutscher KMU aus der Bekleidungsindustrie und aus der Möbelindustrie mit Partnerunternehmen aus Mittel-/Osteuropa. Alle von uns untersuchten Kooperationsbeziehungen existierten mindestens schon seit fünf Jahren. Bei den deutschen KMU handelte es sich vorwiegend um Unternehmen, die in einer Marktnische agierten (z. B. Berufsbekleidung für bestimmte Beschäftigtengruppen, Produktion von Möbeln für Objektausstattungen), oder denen es gelungen war, mit ihren Produkten in großen Handelseinrichtungen gelistet zu werden. ${ }^{6}$ Diese Fallstudien wurden eingebettet in Branchenanalysen in den entsprechenden Ländern, da die Situation der kooperierenden Unternehmen und die Beschaffenheit der jeweiligen Beschäftigungsbedingungen nicht losgelöst von der Entwicklung der Branche insgesamt betrachtet werden können. Die Fallstudien beruhten im Wesentlichen auf ausführlichen thematischen Interviews mit den Eigentümern bzw. den Geschäftsführern und, soweit dies möglich war, mit den betrieblichen Arbeitnehmervertretungen. In der Bekleidungsindustrie gelang es uns darüber hinaus, Interviews mit insgesamt 43 Produktionsarbeiterinnen aus sechs Untersuchungsbetrieben $\mathrm{zu}$ realisieren. ${ }^{7}$ Die betrieblichen Erhebungen wurden ergänzt durch Expertengespräche mit Ge- werkschaftern, Verbandsvertretern und Wissenschaftlern sowie durch die Auswertung statistischer Materialien. Bei den Beschäftigungsbedingungen konzentrierten wir uns auf die Analyse von Löhnen, Arbeitszeiten und Interessenvertretung.

\subsection{ERGEBNISSE: GENERELLE TENDENZEN}

\section{LÖHNE}

Generell kann man feststellen, dass die Löhne in der Bekleidungs- und Möbelindustrie in den von uns einbezogenen mittel-/osteuropäischen Ländern nicht nur signifikant unter dem deutschen Niveau lagen, sondern auch am unteren Ende der Einkommensskala im verarbeitenden Gewerbe in den betreffenden Ländern. Sie haben sich damit, trotz gewisser Lohnerhöhungen in der Möbelindustrie, weitgehend von dem generellen Trend steigender Löhne in diesen Ländern abgekoppelt. Die Löhne, die in den Partnerbetrieben deutscher KMU gezahlt werden, entsprechen im Wesentlichen den geringen Durchschnittslöhnen in den beiden Branchen. Das heißt, eine stabile Kooperationsbeziehung mit einem deutschen Unternehmen kann zwar dazu beitragen, die wirtschaftliche Perspektive der mittel-/osteuropäischen Partnerfirma zu sichern, sie ist aber nicht mit Einkommensvorteilen für die Beschäftigten verbunden. Insbesondere in der Bekleidungsindustrie, zum Teil aber auch in der Möbelindustrie, liegen die Löhne unterhalb des sozialen Minimums zum Erhalt einer Familie. In unseren Mitarbeiterinnenbefragungen in der Bekleidungsindustrie nannten die meisten polnischen Arbeiterinnen einen Betrag zwischen 1.200 und 1.800 PLN (ca. 316-500 €), der notwendig wäre, um mit der Familie einigermaßen über die Runden zu kommen. Ihre rumänischen Kolleginnen hielten im gleichen Zusammenhang eine Summe von 1.000 bis 1.500 RON (ca. 242-424 €) für erforderlich. Angesichts dieser Einkommensverhältnisse spielen familiäre Netzwerke und der Eigenanbau von Lebensmitteln in ländlichen Regionen eine große Rolle, um das Überleben zu sichern. Es liegt auf der Hand, dass alleinerziehende Frauen mit besonders gravierenden Problemen konfrontiert sind. Eine weitverbreitete Praxis in der rumänischen Bekleidungsindustrie besteht in der Ausgabe von Gutscheinen für Lebensmittel durch die Betriebe, die in re- gionalen Supermärkten oder Restaurants eingelöst werden können.

Hinsichtlich der künftigen Entwicklung der Löhne sahen sich die befragten mittel-/osteuropäischen Unternehmer mit einem Dilemma konfrontiert. Auf der einen Seite verwiesen sie darauf, dass niedrige Löhne und Arbeitskosten nach wie vor ein wesentliches Element ihrer Wettbewerbsfähigkeit darstellen. Auf der anderen Seite war sich die Mehrheit der in die Untersuchung einbezogenen Unternehmer und Manager durchaus darüber im Klaren, dass eine auf Dauer praktizierte Niedriglohnstrategie problematische Nebenwirkungen auslöst, die sich auch negativ auf die Leistungsfähigkeit der Unternehmen niederschlagen. Diese Nebenwirkungen manifestieren sich besonders deutlich in einem vielfach beklagten Fachkräftebzw. Arbeitskräftemangel, dem sowohl Migrationsprozesse als auch Abwanderungen in andere Branchen und die nachlassende Bereitschaft junger Leute zu einer entsprechenden Berufsausbildung zugrunde liegen. Eine weitere problematische Auswirkung der niedrigen Löhne besteht offenbar auch im Auftreten von Motivationsproblemen bei den Beschäftigten. Jedenfalls machten mehrere der befragten Unternehmer sowohl in der Möbel- als auch in der Bekleidungsindustrie entsprechende Andeutungen.

Insgesamt gesehen fanden wir in der Bekleidungsindustrie eine größere Differenziertheit von Unternehmenstypen und Beschäftigungsbedingungen als in der Möbelindustrie, wobei diese Differenziertheit auch mit unterschiedlichen Mustern der Funktionsteilung zwischen deutschen und mittel-/osteuropäischen Unternehmen verbunden war. Bei unseren mittel/osteuropäischen Untersuchungsbetrieben in der Möbelindustrie handelte es sich durchweg um strategiefähige Unternehmen, für die zwar die Zusammenarbeit mit

\footnotetext{
Es soll allerdings nicht verschwiegen werden, dass wir auf erhebliche Zugangsprobleme bei den deutschen KMU stießen. Einen möglichen Grund dafür nannte ein deutscher Unternehmer der Bekleidungsindustrie, der sich zu einem Interview bereit erklärte: "Sagen Sie bloß niemanden, dass wir in Polen produzieren lassen. Wenn das unsere Kunden mitkriegen, wollen sie von uns sofort einen Preisnachlass. "

7 Diese Mitarbeiterinnenbefragung wurde durch die Förderung der Friedrich-Ebert-Stiftung ermöglicht und in Zusammenarbeit mit nationalen Partnern realisiert.
} 
deutschen Partnern eine wichtige Rolle spielte, die aber alle über diese Zusammenarbeit hinaus auch eigene Möbel entwarfen, produzierten und eigenverantwortlich auf dem Markt anboten. Dennoch gilt auch für diese Branche, dass die Einkommen der Beschäftigten deutlich unter dem Durchschnitt des verarbeitenden Gewerbes lagen und sich von dem generellen Trend steigender Einkommen in Mittel- und Osteuropa abgekoppelt hatten. Gerade die Situation in der Möbelindustrie macht deutlich, dass Strategiefähigkeit zwar eine notwendige Voraussetzung für wirtschaftliche Leistungskraft und wachsende Verteilungsspielräume ist, dass sie jedoch keinesfalls automatisch zu höheren Löhnen und generell zu besseren Beschäftigungsbedingungen führt. Schwache Gewerkschaften und ein auch auf der Basis von Löhnen ausgetragener Preiswettbewerb stehen der Ausschöpfung dieser gewachsenen Möglichkeiten im Interesse der Arbeitnehmer entgegen. Bei den Untersuchungsbetrieben der Bekleidungsindustrie dominierte der klassische Typ der verlängerten Werkbank, der ausschließlich Produktionsfunktionen für deutsche Partner im Rahmen der Lohnveredlung wahrnahm. Die Löhne waren hier in der Regel extrem niedrig, die wirtschaftliche Lage angespannt. In Rumänien und Polen stießen wir jedoch auch auf Betriebe, denen es gelungen war, komplexere Funktionen des Wertschöpfungsprozesses zu übernehmen.

\section{INTERESSENVERTRETUNG}

Was die Existenz einer betrieblichen Arbeitnehmervertretung betrifft, so bestätigte sich auch in unserem Untersuchungssample die generelle Tendenz, dass derartige Vertretungen in kleineren privaten Betrieben, die nach dem Systemwechsel gegründet wurden, fast völlig fehlen. Eine Arbeitnehmervertretung gab es lediglich in den Fällen, in denen die Unternehmen oder ihre Vorläufer bereits vor 1990 existierten. Die Einstellung der Unternehmer in unseren Untersuchungsbetrieben zu einer betrieblichen Arbeitnehmervertretung war vergleichbar mit der in deutschen KMU (Wassermann/Rhode 2004). Da wo es keine Arbeitnehmervertretung gab, wurde jeder Gedanke an die mögliche Gründung einer derartigen Vertretung meist mit patriarchalischen Argumenten als unnötig und für einen kleineren Betrieb als unangemessen zurückgewiesen. In den Fällen, in denen bereits seit Jahren eine betriebliche Gewerkschaftsorganisation existierte, bemühten sich die Geschäftsführer trotz gelegentlicher Konflikte um eine sachliche kooperative Zusammenarbeit mit dieser. Hier wurden auch Einzelheiten der Arbeitszeit und der Entlohnung in betrieblichen Vereinbarungen geregelt. Die betrieblichen Gewerkschaftsorganisationen spielen auch eine zentrale Rolle bei der Information der Beschäftigten über die Situation und die Perspektiven des Unternehmens. Fehlen sie, unterbleibt eine derartige Information weitgehend. Generell kann man den Führungsstil der Unternehmer in den mittel/osteuropäischen Partnerbetrieben deutscher KMU als eine Kombination von patriarchalischen und autoritären Elementen charakterisieren, wobei fallweise die eine oder die andere Komponente überwiegt.

Die Akteure und Organisationen, die für eine Einhaltung der gesetzlichen Normen auf dem Gebiet des Arbeits- und Sozialrechts sorgen sollen, sind insgesamt wenig effizient. Daraus resultiert eine spezifische Permissivität der Arbeitsbeziehungen und des unternehmerischen Handelns (Bluhm 2006), die auch die systematische Verletzung gesetzlich verbriefter Arbeitnehmerrechte einschließt. Besonders weitverbreitet ist die Praxis, Überstunden auf der gleichen Basis wie Arbeit während der regulären Arbeitszeit zu bezahlen, das heißt, ohne die vom Gesetz vorgeschriebenen Zuschläge. Häufig wird auch der Lohn in einen offiziellen Teil, für den Steuern und Sozialabgaben gezahlt werden, und einen nichtoffiziellen Teil ohne derartige Zahlungen aufgeteilt, was für den Arbeitnehmer Einbußen bei der Zahlung von Krankengeld und von Altersrente zur Folge hat. Obwohl es natürlich immer schwierig ist, im Rahmen sozialwissenschaftlicher Fallstudien derartige Gesetzesverstöße konkret nachzuweisen, liefert doch zumindest unsere Mitarbeiterinnenbefragung in der Bekleidungsindustrie deutliche Indizien dafür, dass auch ein Teil der mittel/osteuropäischen Unternehmen, die im Rahmen von Kooperationsbeziehungen mit deutschen KMU zusammenarbeiten, ein derartiges Verhalten praktiziert. In diesen Fällen ist es sicherlich berechtigt festzustellen, dass die mittel-/osteuropäischen Unternehmer den Flexibilitäts- und Preisforderungen ihrer deutschen Auftraggeber auch dadurch zu entsprechen suchen, dass sie gegen gesetzlich verbriefte Arbeitnehmerrechte verstoßen.

\section{Funktionsmechanismen und Entwicklungstenden- zen der Kooperations- beziehungen}

\subsection{DIE ROLLE DER DEUTSCHEN PARTNERUNTERNEHMEN}

Aus den Schilderungen der mittel-/osteuropäischen Unternehmer, aus Interviews mit den Geschäftsführern der deutschen Partnerunternehmen, soweit wir sie durchführen konnten, sowie aus dem Abgleich mit bereits vorliegenden Forschungsergebnissen zu grenzüberschreitenden Kooperationsbeziehungen (Lungwitz/Preusche 2002; Wannöffel et al. 2003) ergibt sich ein relativ konsistentes Bild hinsichtlich des praktischen Funktionierens dieser Beziehungen und der Rolle der deutschen Partnerunternehmen in diesem Rahmen. Wie bereits eingangs erwähnt, ist das Kostenmotiv für deutsche KMU im gegebenen Zusammenhang von entscheidender Bedeutung. Konkret bedeutet dies, dass die deutschen Unternehmer in erster Linie an niedrigen Preisen für die Lieferungen ihrer mittel-/osteuropäischen Kooperationspartner interessiert sind, wobei selbstverständlich vorausgesetzt wird, dass die Unternehmen aus Mittel-/Osteuropa auch in der Lage sind, die Anforderungen hinsichtlich Qualität, Flexibilität und Liefertreue zu erfüllen. Kooperationsbeziehungen entwickeln sich nur dann, wenn sich die Partner wechselseitig von ihrer Kooperationsfähigkeit und von ihrer Bereitschaft überzeugt haben, die Anforderungen der Kooperation zu erfüllen und den Partner schädigendes Verhalten zu unterlassen. Aus Sicht der deutschen Unternehmer verläuft die Genese einer derartigen Beziehung in der Regel so, dass sich die initiierende Seite zunächst durch einen Besuch bei einem potenziellen Kandidaten und durch die Vergabe von Probeaufträgen von dessen grundsätzlicher Kooperationsfähigkeit überzeugt. Sind die Erfahrungen positiv, wird die Zusammenarbeit allmählich ausgeweitet. Das Vertrauen, dass der Partner auch bereit ist, die Anforderungen der Zusammenarbeit zu erfüllen, stützt sich in erster Linie auf das Wissen, dass das mittel-/osteuropäische Unternehmen auf diese Zusammenarbeit angewiesen ist. Ist die Zusammenarbeit erst einmal etabliert 
und verläuft aus Sicht des deutschen Unternehmers positiv, zieht eine gewisse Routine in die Koordinierung der Kooperationsbeziehung ein. Dieser durch Routine und den hohen Stellenwert persönlicher Beziehungen geprägte Koordinationsmodus kommt den Besonderheiten kleiner und mittlerer Unternehmen entgegen, da er mit relativ geringen Transaktionskosten verbunden ist und an Praktiken anknüpft, die auch innerhalb der Unternehmen existieren. Von dem mittel-/osteuropäischen Unternehmer wird erwartet, dass er in seinem Unternehmen die Voraussetzungen dafür schafft, dass die Lieferungen stabil zu niedrigen Preisen und in angemessener Qualität erfolgen können, ohne dass sich der deutsche Unternehmer hier einmischt. Dieser spezifische Koordinationsmodus ist der Grund dafür, dass sich der deutsche Partner für solche „Details“ wie Löhne, Arbeitszeit, Arbeitsbeziehungen oder die Einhaltung von gesetzlichen Bestimmungen in seinem mittel-/osteuropäischen Kooperationsunternehmen in der Regel nicht interessiert.

Die von uns untersuchten Kooperationsbeziehungen existierten bereits seit mindestens fünf Jahren, einige von ihnen sogar schon wesentlich länger, sodass man von einer gewissen Stabilität dieser Beziehungen sprechen kann. Dies ist durchaus nicht selbstverständlich, bedenkt man den wachsenden Preis- und Kostendruck, dem deutsche KMU als Nischenanbieter oder als Lieferanten für Großunternehmen unter den Bedingungen der Globalisierung ausgesetzt sind und der auch für diese Unternehmen einen Anreiz schafft, die bestehende Struktur ihrer Wertschöpfungskette kritisch zu hinterfragen. Deutsche KMU, die Kooperationsbeziehungen etwa mit polnischen oder tschechischen Partnerunternehmen unterhalten, haben durchaus auch die Möglichkeit, diese Beziehungen zu beenden und noch preisgünstigere Partner in Ländern wie beispielsweise der Ukraine zu finden. Es gibt mit Sicherheit deutsche KMU, die eine derartige Umgestaltung ihrer Wertschöpfungskette vollzogen haben oder Entsprechendes planen. Andererseits zeigt sich jedoch auch in unserem Sample sehr deutlich, dass die KMU eine eingespielte Kooperationsbeziehung mit einem mittel-/osteuropäischen Partnerunternehmen, mit der sie grundsätzlich zufrieden sind, nicht vorschnell zur Disposition stellen. Die Suche nach einem neuen Partner birgt zwar Chancen, aber auch massive Risiken und verlangt höhere Transaktionskosten für die Etablierung der Zusammenarbeit. Mit wachsender regionaler Entfernung gilt dies auch für den Transport der bezogenen Güter. „Wir werden entweder gemeinsam die Zukunft meistern oder wir gehen zusammen unter", charakterisierte ein befragter deutscher Unternehmer der Bekleidungsbranche, der Lohnveredlung durch einen polnischen Partner betreibt, die Situation. Wir hatten auch zumindest bei unseren Untersuchungen in Polen den Eindruck, dass die befragten Unternehmer die Gefahr eines Wechsels ihrer deutschen Auftraggeber zu einem noch preiswerteren, aber weiter „im Osten“ gelegenen Kooperationspartner geringer einschätzten als noch vor wenigen Jahren. Durchaus selbstbewusst wurde auf die eigene Qualitätsfähigkeit, auf den Vorteil regionaler Nähe zu Deutschland und, was die Möbelindustrie betrifft, auf die deutlich verbesserte technische Ausstattung der Unternehmen verwiesen. Die deutschen Firmen, die einen derartigen Wechsel anstrebt, hätten ihn bereits vollzogen, bemerkte eine polnische Unternehmerin aus der Bekleidungsbranche. Eher sahen die tschechischen und polnischen Unternehmer die Gefahr darin, dass die deutschen KMU zu anderen Partnern in ihren Ländern wechseln könnten, die die entsprechenden Waren zu einem noch niedrigeren Preis liefern.

\subsection{PERSPEKTIVEN GRENZÜBER- SCHREITENDER KOOPERATIONEN UND EINER EUROPÄISCHEN ARBEITSTEILUNG}

Die mittel-/osteuropäischen Kooperationspartner deutscher KMU sehen sich einem starken Kostendruck ausgesetzt, der im Wesentlichen aus zwei Quellen resultiert. Zum einen versuchen ihre deutschen Auftraggeber, die sich oft selbst in einer schwierigen wirtschaftlichen Situation befinden und einem starken Preiswettbewerb ausgesetzt sind, Druck auf die Preise ihrer Partnerunternehmen in Mittel- und Osteuropa auszuüben. Zum anderen gibt es seit Jahren eine stabile Aufwertungstendenz der mittel-/osteuropäischen Währungen gegenüber dem Euro. Im Prinzip müssten die mittel-/osteuropäischen Firmen darauf mit Preiserhöhungen gegenüber ihren deutschen Auftraggebern reagieren, was sie jedoch aufgrund der gegebenen Machtrelation vielfach als nicht durchsetz- bar betrachten. Auch daraus resultiert ein starker Zwang, die entsprechenden Verluste durch Kostensenkungen zu kompensieren. Angesichts dieser Situation versuchen die mittel-/osteuropäischen Unternehmen im Rahmen ihrer Möglichkeiten, die Kosten zu senken. Während bei den Betrieben der Möbelindustrie hier Konzepte der technischen Modernisierung und Produktivitätserhöhung eine wichtige Rolle spielen, fanden wir in unserem Sample sowohl polnische als auch rumänische Betriebe der Bekleidungsindustrie, die ihrerseits im Begriff waren, Näharbeiten in die Ukraine bzw. nach Moldawien auszulagern. Von den deutschen Partnern werden solche Auslagerungen durchaus begrüßt, wenn der polnische bzw. rumänische Partnerbetrieb die Einhaltung der Qualitätsstandards garantiert.

Diese Programme zur Kostenreduktion werden jedoch nur dann zu einer dauerhaften wirtschaftlichen Entwicklung führen, wenn es gelingt, die Arbeitsteilung zwischen Unternehmen aus West- und Osteuropa grundsätzlich anders zu strukturieren. Das traditionelle Muster der Arbeitsteilung zwischen deutschen KMU und ihren mittel-/osteuropäischen Kooperationspartnern besteht darin, dass Letzteren vor allem arbeitsintensive und eher geringe Qualifikationen erfordernde Prozessphasen zur Realisierung übertragen werden, während alle strategischen Funktionen in der Wertschöpfungskette, wie Entwicklung und Vermarktung der Erzeugnisse, aber auch wertschöpfungsintensive Produktionsphasen dem deutschen Partner vorbehalten bleiben.

Einen besonders deutlichen und zugespitzten Ausdruck findet dieses Muster der Arbeitsteilung in der passiven Lohnveredlung in der Bekleidungsindustrie. Diese Art der Arbeitsteilung führt in den mittel-/osteuropäischen Unternehmen zwangsläufig zu geringer Wertschöpfung, niedrigen Gewinnen, eingeschränkten Spielräumen für die Modernisierung und zu niedrigen Löhnen. Die Anreize für die Unternehmer, das Überleben ihrer Firmen auch durch die Umgehung von Normen des Arbeits- und Sozialrechts zu gewährleisten, sind hoch. Es liegt auf der Hand, dass das Ziel der Europäischen Union, eine allmähliche Konvergenz der Lebensbedingungen zwischen ihren Mitgliedsländern zu erreichen, auf einer derartigen Grundlage nicht einzulösen ist. Obwohl diese Art der Arbeitsteilung nach wie vor eine bedeutende Rolle spielt, 
waren auch in den beiden von uns untersuchten Branchen deutlich darüber hinausweisende Entwicklungstendenzen feststellbar. Sie sind grundsätzlich darauf gerichtet, den mittel-/osteuropäischen Firmen komplexere, mit höherer Wertschöpfung verbundene Aufgaben zuzuweisen und sie auch stärker in strategische Funktionen der Wertschöpfungskette zu integrieren. Dabei sei es dahingestellt, ob sich bestehende Kooperationsbeziehungen in diese Richtung entwickeln oder ob neue Beziehungen auf einer von vornherein anders strukturierten Grundlage entstehen. In der Bekleidungsindustrie würde dies beispielsweise den Übergang von der Lohnveredlung $\mathrm{zu}$ einer „ready-to-sell“ Produktion für westeuropäische Auftraggeber bedeuten, inklusive der Verantwortung für Design und Logistik, oder den Versuch, zunächst auf den mittel-/osteuropäischen Märkten eigene Produkte in eigenen Vertriebseinrichtungen abzusetzen. In der Möbelindustrie verbinden sich diese Bestrebungen mit einem wachsenden Stellenwert der Lieferung von Komplettmöbeln an deutsche Partner anstelle der Lieferung von Komponenten und ebenfalls mit dem Versuch, stärker selbst entwickelte Möbel eigenverantwortlich auf in- und ausländischen Märkten abzusetzen.

Was die Lohnentwicklung betrifft, so bietet eine wachsende Wertschöpfung in den mittel-/osteuropäischen Unternehmen zwar die Chance für weitere Lohnerhöhungen, diese werden sich aber aufgrund der gegebenen Rahmenbedingungen in erster Linie an den Zwängen des Arbeitsmarktes orientieren. Gefragte Fachkräfte können sicher mit wachsenden Einkommen rechnen, für die anderen Beschäftigten werden die Verbesserungen eher gering ausfallen. Die Entwicklung von Arbeitnehmervertretungen insbesondere im Bereich mittel-/ osteuropäischer KMU sowie die generelle Stärkung gewerkschaftlicher Strukturen bleiben erstrangige Voraussetzungen dafür, dass die Chancen der wirtschaftlichen Entwicklung auch den Arbeitnehmern zugutekommen. Bisher war für die meisten der neuen EU-Staaten eine negative Verteilungsbilanz charakteristisch, was auf ein
Zurückbleiben des lohnpolitischen Aufholprozesses gegenüber den wirtschaftlichen Möglichkeiten verweist (Schulten 2006).

Es deutet einiges darauf hin, dass die Entwicklung grenzüberschreitender Kooperationsbeziehungen deutscher KMU künftig noch stärker zu der ohnehin bereits sehr ausgeprägten sozialen Differenzierung innerhalb Mittel- und Osteuropas beitragen wird. Es wird auf der Ebene der mittel-/osteuropäischen Partnerfirmen Gewinner geben, die von der sich abzeichnenden neuen Arbeitsteilung profitieren können. Und es wird Verlierer geben, die versuchen, ihre Existenz auch künftig auf der Übernahme arbeitsintensiver Teilprozesse für deutsche Partner zu gründen, und die niedrige Löhne und eine weitgehende Unterordnung der Beschäftigten unter die Flexibilitätsanforderungen des Unternehmens dafür als notwendige Voraussetzung betrachten. Aber auch innerhalb der „Gewinnerfirmen" werden aufgrund des Fehlens einer solidarischen Lohnpolitik die sozialen Unterschiede zwischen einzelnen Beschäftigtengruppen wohl größer werden. 
Bluhm, K. (2000): East-West Integration and the Changing German Production Regime: A Firm-Centered Approach. www.ces.fas.harvard.edu/publications/Bluhm53.pdf Bluhm, K. (2006): Auflösung des Liberalisierungsdilemmas, in: Berliner Journal für Soziologie 2, S. 173-188

Brandl, S./Stelzl, B. (2005): Internationale Arbeitsbeziehungen - Globalisierung als Chance für die deutschen Gewerkschaften?, in: WSI-Mitteilungen 2, S. 82-89

Carley, M. (2006): Working time developments - 2005, EIRO, Dublin European Commission (2004): Industrial Relations in Europe, Brüssel Faust, M./Voskamp, U./Wittke, V. (Eds.) (2004): European Industrial Restructuring in a Global Economy: Fragmentation and Relocation of Value Chains, Göttingen

Gardawski, J. (2005): Polnisches Zentrum zur Überwachung der industriellen Beziehungen, Warszawa

Gohde, H. (2005): Betriebliche Interessenvertretungen in kleinen und mittleren Unternehmen der Länder Frankreich, Italien, Österreich, Schweden und Ungarn, Studie im Auftrag der Hans-Böckler-Stiftung, Düsseldorf Golinowska, S. (1997): Polska bieda II - Kryteria, oocena, przeciwdziałanie, IIPiSS, Warszawa

Greven, T. (2006): Auf dem Prüfstand: Gewerkschaftsstrategien zur Regulierung globaler Konkurrenz, in: WSI-Mitteilungen 1, S. 10-15 IPiSS (2006): Wysokość minimum socjalnego w latach 1990-2005, http://www.ipiss.com.pl/badania/minimum/-wer2.pdf

Kinkel, S./Lay, G./Maloca, S. (2004): Produktionsverlagerungen ins Ausland und Rückverlagerungen, Forschungsbericht, Fraunhofer-Institut für Systemtechnik und Innovationsforschung, Karlsruhe

Kurz, C./Wittke, V. (1998): Die Nutzung industrieller Kapazitäten in Mittelosteuropa durch westliche Unternehmen - Entwicklungspfade einer neuen industriellen Arbeitsteilung, in: SOFI-Mitteilungen 26, S. 45-68
Lungwitz, R.-E./Preusche, E. (2002): Kooperation zwischen kleinen und mittelständischen Firmen in Ostdeutschland und Firmen in Polen und der Tschechischen Republik, Projektbericht an die DFG, WISOC, Chemnitz Musiolek, B. (2006): Interessenvertretungen in der Bekleidungsindustrie Mittel- und Südosteuropas, in: WSI-Mitteilungen 1, S. 47-51

Peplak, T. (2002): Zur Lage der polnischen Textil- und Bekleidungsindustrie http://www.fashionet.pl/?sid=view\&news=257

Preiss, T. (2005): Mehr als Produktion gefragt. Textil- und Bekleidungsindustrie: Nach wie vor gute Kooperationschancen für deutsche Unternehmen, in: Ost-West-Contact 10, S. 48-49

Schulten, T. (2004): Europäischer Tarifbericht des WSI 2003/2004, in: WSI-Mitteilungen 7, S. 347-357

Schulten, T. (2006): Europäischer Tarifbericht des WSI 2005/2006, in: WSI Mitteilungen 7, S. 365-373

Semlinger, K. (1993): Effizienz und Autonomie in Zulieferungsnetzwerken - Zum strategischen Gehalt von Kooperation, in: Sydow, J./Staehle, W. H. (Hrsg.): Managementforschung 3, Berlin, New York, S. 310-353

Towalski, R. (2006): Employment relations in SME - The Case of Poland, EIRO-Foundation, Dublin

Wassermann, W./Rhode, W. (2004): Konfliktfeld Kleinbetrieb, Mittelstand zwischen Alleinherrschaft und Mitbestimmung, Frankfurt a. M. Wannöffel, M./Le, P./Campagna, S. (2003): Prozesse, Probleme und Praktiken der internationalen Kooperation zwischen kleinen und mittleren Unternehmen, in: Hirsch-Kreinsen, H./Wannöffel, M. (Hrsg.): Netzwerke kleiner Unternehmen, Berlin, S. 207-227

Welzmüller, R. (2004): EU-Osterweiterung, IGM, Blaue Reihe, Frankfurt a. $M$. 